\title{
Estudo comparativo entre estibogluconato de sódio BP 88® e antimoniato de meglumina no tratamento da leishmaniose cutânea II. Toxicidade bioquímica e cardíaca
}

\author{
Comparative study between sodium stibogluconate BP $88 \AA$ and meglumine \\ antimoniate in cutaneous leishmaniasis treatment. \\ II. Biochemical and cardiac toxicity
}

\begin{abstract}
Ana Cristina R. Saldanha', Gustavo Adolfo Sierra Romero', Conceição Guerra², Edgar Merchan-Hamann ${ }^{3}$ e Vanize de Oliveira Macedo ${ }^{1}$
\end{abstract}

\begin{abstract}
Resumo Foi avaliada a toxicidade de dois antimoniais pentavalentes em 111 pacientes com leishmaniose cutânea. Quarenta e sete pacientes receberam antimoniato de meglumina (Grupo I) e 64 pacientes, estibogluconato de sódio BP $88 \AA$ (Grupo II), $20 \mathrm{mg} \mathrm{Sb} / \mathrm{kg} / \mathrm{dia}$ por 20 dias. Realizou-se a avaliação de aminotransferases, fosfatase alcalina, amilase, creatinina, uréia, exame de urina e eletrocardiograma, antes do tratamento e no décimo e vigésimo dias. Observou-se maior freqüência de valores anormais de aminotransferases no décimo e vigésimo dias de tratamento no grupo II $(p<0,001)$ e maior proporção de valores anormais de amilase no décimo dia no mesmo grupo $(p<0,001)$. Houve maior variação dos níveis de aminotransferases, fosfatase alcalina e amilase nos primeiros dez dias no grupo II $(p<0,01)$. No vigésimo dia observou-se maior variação nos níveis de aminotransferases no grupo II ( $p=0,02$ e $p=0,03$, respectivamente). Quarenta e três porcento dos pacientes do grupo I e 54\% dos pacientes do grupo Il apresentaram alterações eletrocardiográficas $(p=0,30)$.
\end{abstract}

Palavras-chaves: Leishmaniose cutânea. Estibogluconato de sódio. Antimoniato de meglumina. Toxicidade.

Abstract Toxicity of two antimonial pentavalents were evaluated in 111 patients with cutaneous leishmaniasis. Forty seven patients received meglumine antimoniate (Group I) and 64 patients, sodium stibogluconate BP $88 \AA$ (Group II), $20 \mathrm{mg} \mathrm{Sb} / \mathrm{kg} /$ day for 20 days. Evaluation of aminotransferases, alkaline phosphatase, amilase, creatinine, urea, urine analysis and electrocardiogram were performed at baseline, on the tenth and twentieth day of treatment. Greater frequency of aminotransferase abnormal levels were observed on the tenth and twentieth days in group II $(p<0,001)$ and a greater proportion of amilase abnormal levels at the tenth day in the same group $(p<0,001)$. There was a greater variation of aminotransferases, alkaline phosphatase and amilase in the first ten days of treatment in group II $(p<0,01)$. On the twentieth day there was a greater variation of aminotransferase levels in group $I /(p=0,02$ and $p=0,03$, respectively). Forty three percent of group I and $54 \%$ of group II showed electrocardiographic abnormalities $(p=0,30)$.

Key-words: Cutaneous leishmaniasis. Sodium stibogluconate. Meglumine antimoniate. Toxicity.

\footnotetext{
1. Núcleo de Medicina Tropical da Universidade de Brasília, Brasília, DF; 2. Laboratório Central de Saúde Pública, Prof. Gonçalo Moniz, Salvador, BA; 3. Departamento de Saúde Coletiva da Faculdade de Ciências da Saúde da Universidade de Brasília, Brasília, DF. Endereço para correspondência: Drª Ana C.R. Saldanha. Núcleo de Medicina Tropical/UnB, Campus Universitário Asa Norte, 70919970 Brasília, DF, Brasil.

Fax: $5561273-2811$.

e-mail: tropical@unb.br

Recebido para publicação em 8/2/99.
} 
Os antimoniais pentavalentes produzem efeitos tóxicos caracterizados principalmente por alterações eletrocardiográficas, das provas de função hepática, pancreática e renal. Mialgias, artralgias e febre são os efeitos colaterais comuns e todos freqüentemente são dependentes da dose e do tempo de tratamento 26789 .

O presente estudo teve como objetivo comparar a toxicidade hepática, pancreática, renal e cardíaca produzida pelo antimoniato de meglumina (Rhodia, São Paulo, Brasil) e o estibogluconato de sódio BP 88® (Shandong Xinhua, China) em pacientes com leishmaniose cutânea (LC) na área endêmica do Distrito de Corte de Pedra, Presidente Tancredo Neves, Bahia, Brasil, durante um estudo experimental aberto realizado para comparar a eficácia dos dois compostos ${ }^{14}$. Nesta região ocorre transmissão predominante de Leishmania (Viannia) braziliensis ${ }^{12}$.

\section{PACIENTES E MÉTODOS}

Foram incluídos 111 pacientes de 7 a 60 anos de idade, com LC, atendidos no Centro de Saúde de Corte de Pedra que receberam tratamento com antimoniato de meglumina ou estibogluconato de sódio BP $88 \AA$ durante um ensaio clínico para avaliar a eficácia destas drogas. Foram excluídos os pacientes que referiram tratamento prévio com antimoniais pentavalentes ou drogas leishmanicidas nos últimos seis meses, evidência clínica de alterações cardíacas, tuberculose, hanseníase, câncer, diabetes mellitus, hipertensão arterial sistêmica não controlada, comprometimento vascular periférico, história de alcoolismo, tratamento com corticosteróides ou outro imunossupressor e grávidas.

Quarenta e sete pacientes receberam tratamento com antimoniato de meglumina (Rhodia, São Paulo, Brasil. Lote de no 059L06) e constituíram o grupo I. Sessenta e quatro pacientes receberam tratamento com estibogluconato de sódio BP $88 \AA$ (Shandong, Xinhua, China. Lote de no 96208) constituindo o grupo II. Em ambos os grupos utilizou-se a dose de $20 \mathrm{mg}$ de $\mathrm{Sb} / \mathrm{kg} /$ dia, aplicação única diária, durante 20 dias, via intravenosa ou intramuscular, não diluída e administrada lentamente. Ambas as drogas foram acondicionadas em geladeira a $4^{\circ} \mathrm{C}$.

Avaliação da toxicidade renal, hepática e pancreática. Foram colhidas amostras de soro antes do tratamento e no décimo e vigésimo dias. Os ensaios foram realizados de maneira automatizada no Serviço de Bioquímica, Unidade de Biologia Médica do Laboratório Central (LACEN), Bahia, no período de fevereiro a maio de 1997 utilizando-se aparelho Cobas Mira Plus (Roche) e os métodos descritos na Tabela 1. Para complementar a avaliação da toxicidade renal coletou-se uma amostra da urina de cada paciente com a mesma periodicidade. Estas amostras foram análisadas com fita colorimétrica COMBUR 10 Test ${ }^{\circledR}$ M (Boehringer Mannheim), que permitiu determinar densidade urinária, $\mathrm{pH}$, presença de leucócitos, nitritos, proteínas, glicose, corpos cetônicos, urobilinogênio, bilirrubinas hemácias e hemoglobina.

Tabela 1 - Métodos utilizados na avaliação bioquímica.

\begin{tabular}{|c|c|c|c|}
\hline Prova & Método & Marca & Valor de referência \\
\hline Uréia & Urease & Wiener & 10 a $50 \mathrm{mg} / \mathrm{dl}$ \\
\hline Creatinina & Jaffé cinético & Laborlab & 0,4 a $1,3 \mathrm{mg} / \mathrm{dl}$ \\
\hline AST & Cinético UV & Wiener & 12 a $46 U / I$ \\
\hline ALT & Cinético UV & Wiener & 3 a $50 U / 1$ \\
\hline Fosfatase alcalina & Cinético UV & Wiener & ${ }^{*} \mathrm{~A}: 65$ a $300 \mathrm{U} /{ }^{*} \mathrm{C}:$ até $645 \mathrm{U} / \mathrm{I}$ \\
\hline Amilase & Cinético UV & Wiener & até 220U/l \\
\hline
\end{tabular}

${ }^{\star} \mathrm{A}=$ adulto $; \mathrm{C}=$ criança

Avaliação da toxicidade cardíaca. A toxicidade cardíaca foi avaliada com eletrocardiogramas antes do tratamento, no décimo e vigésimo dias do esquema terapêutico e três meses após o tratamento. Foi utilizado um aparelho Hewlet Packard modelo 1500. Os traçados foram realizados por um técnico previamente treinado, seguindo os critérios de padronização dos estudos longitudinais da Doença de Chagas ${ }^{3}$. Cada traçado foi identificado com um número aleatório de uma lista preparada no programa Epi-Info, versão 6.04 para serem avaliados de forma mascarada em relação ao grupo de intervenção e à sequência cronológica. Foram 
seguidas as normas de interpretação descritas pelo código de Minnesota para a avaliação das alterações do QT e ST ${ }^{13}$.

Os dados foram analisados no programa EPIInfo, versão 6.04. Para todas as análises foi fixado um limite de significância igual a 0,05. Para comparar proporções foi utilizado o teste do chiquadrado e T de student para comparar médias.

O estudo cumpriu com os requisitos da Resolução do Conselho Nacional de Saúde, 196/ 96 e foi aprovado pelo Comitê de ética em pesquisa envolvendo seres humanos da Faculdade de Ciências da Saúde da Universidade de Brasília.

\section{RESULTADOS}

Foram analisados dados dos 111 pacientes que realizaram avaliação sérica nos dia zero, 10 e 20 do tratamento. Os pacientes dos grupos I e II não diferiram em relação à média de idade, distribuição por sexo, número e localização das lesões e o tempo de evolução da doença. A análise qualitativa entre os dois grupos de tratamento baseando-se na comparação de proporções de valores acima do normal aparece na Tabela 2.

Tabela 2 - Análise qualitativa da freqüência de alterações enzimáticas nos dois grupos do estudo em relação ao dia de tratamento.

\begin{tabular}{|c|c|c|c|c|c|c|}
\hline \multirow{3}{*}{ Enzima } & \multirow{2}{*}{\multicolumn{2}{|c|}{$\begin{array}{c}\text { Dia } 10 \\
\% \text { alterados }\left(\mathrm{VM}^{*}\right)\end{array}$}} & \multirow{3}{*}{$\mathrm{p}^{\star *}$} & \multicolumn{2}{|c|}{ Dia 20} & \multirow{3}{*}{$\mathrm{p}^{* *}$} \\
\hline & & & & \multicolumn{2}{|c|}{$\%$ alterados $\left(\mathrm{VM}^{*}\right)$} & \\
\hline & grupo I & grupo II & & grupol & grupo II & \\
\hline AST & $15,0(122)$ & $72,6(317)$ & $<0,001$ & $19,0(130)$ & $59,0(1268)$ & $<0,001$ \\
\hline ALT & $2,0(79)$ & $46,8(327)$ & $<0,001$ & $6,3(106)$ & $31,5(981)$ & 0,001 \\
\hline Fosfatase alcalina & $8,5(396)$ & $8,0(612)$ & 0,80 & $14,8(404)$ & $6,2(572)$ & 0,23 \\
\hline Amilase & $36,0(896)$ & $71,5(1020)$ & $<0,001$ & $22,0(1108)$ & $41.0(4176)$ & 0,06 \\
\hline
\end{tabular}

*Valor máximo detectado em $\mathrm{U} / \mathrm{L}$ ** Chi-quadrado

Análise quantitativa entre os pacientes de um mesmo grupo (intra-grupo) e entre os dois grupos de tratamento (inter-grupo). Para cada sistema enzimático foi aferida a média das diferenças entre os valores obtidos no dia 10 e aqueles obtidos no dia zero (valor do dia 10 - valor do dia $0=\mathrm{Di} 1$ ). O mesmo foi realizado com as diferenças obtidas entre os dias 20 e 10 (dia 20 - dia $10=\mathrm{Di} 2$ ) e entre os dias 20 e zero (dia 20 - dia $0=\mathrm{Di} 3$ ). Estes dados serviram de base para a análise realizada entre as médias das diferenças obtidas nos dois grupos. Quando comparadas as médias das diferenças dos valores enzimáticos, nos dias 10 e zero, observou-se aumento estatisticamente significativo do valor de todas as enzimas no grupo II. $\mathrm{Na}$ análise das médias das diferenças dos dias 20 e 10, observou-se que a variação foi estatisticamente significativa, para a fosfatase alcalina, cujo valor médio esteve mais elevado no grupo I. Entre os dias 20 e zero houve uma elevação dos níveis de todas as enzimas existindo diferença estatisticamente significativa nos valores de AST e ALT, que foi mais expressiva no grupo II (Tabela 3).

Análise das alterações eletrocardiográficas. Foram registradas as alterações eletrocardiográficas que apareceram a partir do décimo dia de tratamento. Vinte (43\%) pacientes do grupo I e $28(54 \%)$ pacientes do grupo II apresentaram alguma alteração durante o tratamento $\left(x^{2}=1,05\right.$; $p=0,30$ ). Foram observadas alterações isquêmicas em $11 \%$ dos pacientes do grupo I e em $27 \%$ do grupo II ( $\left.p=0,04 ; x^{2}=4,12 ;\right)$. Observaram-se alterações desenvolvidas durante o tratamento que persistiram até o terceiro mês de acompanhamento em 10 pacientes (Tabela 4).

Correlação entre a toxicidade hepática, pancreática e as alterações eletrocardiográficas. Dos 26 pacientes que tiveram alterações eletrocardiográficas no grupo I, 12 (46\%) apresentaram alterações das enzimas hepáticas e/ou pancreáticas durante o tratamento. Em dois pacientes não foi realizada a avaliação bioquímica. Dos 28 pacientes do grupo II, que apresentaram alguma alteração eletrocardiográfica, 24 (85\%) apresentaram elevação dos níveis de enzimas hepáticas e/ou pancreáticas. A freqüência de alterações eletrocardiográficas associadas à elevação dos níveis de enzimas hepáticas e/ou pancreáticas foi maior no grupo II $\left(x^{2}=9,42 ; p=\right.$ 0,02 ). Durante a realização do tratamento 58 pacientes queixaram-se de palpitações, anorexia e/ou dor abdominal. Em 13 (22,4\%) desses indivíduos houve elevação concomitante dos 
níveis séricos de amilase e/ou transaminases, associada a alteração do traçado eletrocardiográfico. Destes 13 casos, $77 \%$ pertenciam ao grupo II e $23 \%$ ao grupo I $\left(x^{2}=7,54 ; p=0,006\right)$.
Não ocorreram alterações nos níveis séricos de creatinina e uréia, nas densidades urinárias, no $\mathrm{pH}$ urinário e não se observaram proteinúria, hematúria ou leucocitúria.

Tabela 3 - Análise intra e intergrupos das médias das diferenças e das variações dos valores enzimáticos nos dois grupos terapêuticos.

\begin{tabular}{|c|c|c|c|}
\hline \multirow[b]{2}{*}{ Enzimas avaliadas } & \multicolumn{3}{|c|}{$\begin{array}{l}\text { Média das diferenças } \\
\text { (espectro total de variação das diferenças em U/L) }\end{array}$} \\
\hline & $\begin{array}{c}\text { Di1 } \\
\text { (D10- D0) }\end{array}$ & $\begin{array}{c}\mathrm{Di2} \\
(\mathrm{D} 20-\mathrm{D} 10)\end{array}$ & $\begin{array}{c}\text { Di3 } \\
\text { (D20- D0) }\end{array}$ \\
\hline \multicolumn{4}{|l|}{ Grupo I $(N=47)$} \\
\hline AST & $8,7(4,1 ; 13.2)$ & $1,5(-4,7 ; 7,8)$ & $10,2(4,3 ; 16,1)$ \\
\hline ALT & $6,4(3,4 ; 9,5)$ & $-4,0(-0,1 ; 9,8)$ & $11,42(6,6 ; 16,3)$ \\
\hline Fosfatase alcalina & $-21,3(-35,4 ;-7,2)$ & $18,2(6,8 ; 29,7)$ & $-3,0(-14,0 ; 7,9)$ \\
\hline Amilase & $75,6(29,6 ; 121,7)$ & $68,5(31,5 ; 105,6)$ & $144,2(90,2 ; 198,2)$ \\
\hline \multicolumn{4}{|l|}{ Grupo II (N = 64) } \\
\hline AST & $66,4(50,0 ; 83,0)$ & $-4,2(-46,9 ; 38,5)$ & $61,2(22,6 ; 99,9)$ \\
\hline ALT & $54,2(39,0 ; 69,3)$ & $4,9(-36,4 ; 28,6)$ & $50,4(19,4 ; 81,4)$ \\
\hline Fosfatase alcalina & $24,4(-2,6 ; 51,3)$ & $-3,4(-19,7 ; 12,9)$ & $20,9(-6,6 ; 48,5)$ \\
\hline Amilase & $151,3(113,6 ; 189,1)$ & $38,6(-88,9 ; 166,1)$ & $190,2(66,1 ; 314,2)$ \\
\hline \multicolumn{4}{|l|}{ Diferenças intergrupos ${ }^{*}$} \\
\hline AST & $<0,01$ & 0,82 & 0,02 \\
\hline ALT & $<0,01$ & 0,59 & 0,03 \\
\hline Fosfatase alcalina & $<0,01$ & 0,04 & 0,16 \\
\hline Amilase & 0,01 & 0,69 & 0,55 \\
\hline
\end{tabular}

* Valor de $\mathrm{p}$ (T de student)

Tabela 4 - Distribuição das alterações eletrocardiográficas em relação ao grupo terapêutico e o dia de acompanhamento clínico.

\begin{tabular}{|c|c|c|c|c|c|c|}
\hline \multirow{3}{*}{ Alterações } & \multicolumn{3}{|c|}{ Grupo I } & \multicolumn{3}{|c|}{ Grupo II } \\
\hline & dia 10 & dia 20 & dia 110 & dia 10 & dia 20 & $\operatorname{dia} 110$ \\
\hline & $(\mathrm{N}=46)$ & $(\mathrm{N}=46)$ & $(\mathrm{N}=39)$ & $(\mathrm{N}=52)$ & $(N=51)$ & $(N=40)$ \\
\hline Aumento do intervalo $\mathrm{QT}\left(\mathrm{Qt}_{\mathrm{c}}{ }^{*}\right)$ & 6 & 5 & 0 & 4 & 4 & 2 \\
\hline Isquemia subepicárdica & 2 & 3 & 0 & 5 & 7 & 2 \\
\hline $\begin{array}{l}\text { Alterações primárias da } \\
\text { repolarização ventricular }\end{array}$ & 2 & 1 & 0 & 1 & 2 & 1 \\
\hline Bigeminismo & 0 & 0 & 0 & $1^{* * *}$ & 0 & 0 \\
\hline Outras $^{* *}$ & 3 & 4 & 2 & 4 & 6 & 3 \\
\hline
\end{tabular}

* Intervalo QT corrigido. ${ }^{*}$ Arritmia sinusal; bradiarritmia sinusal; extrassístoles isoladas; ritmo juncional e bloqueio AV $1^{\circ} \mathrm{grau} .{ }^{* \star *} \mathrm{O}$ tratamento foi suspenso no décimo dia.

\section{DISCUSSÃO}

Observou-se que a elevação dos níveis enzimáticos de AST, ALT e amilase ocorreu principalmente durante a primeira metade do tratamento. Estas alterações foram mais freqüentes no grupo tratado com estibogluconato de sódio. Os níveis séricos de fosfatase alcalina não mostraram alterações significativas em nenhum dos dois grupos. Apesar da variação dos valores enzimáticos ter sido menor na segunda metade do tratamento, valores de amilase tão elevados quanto 4000U/I detectaram-se neste período em um indivíduo tratado com estibogluconato de sódio. O valor máximo de amilase no grupo tratado com antimoniato de meglumina foi $1108 \mathrm{U} / \mathrm{l}$. Na maioria dos pacientes a elevação dos níveis séricos de amilase foi assintomática. De quatro pacientes que apresentaram níveis de amilase acima de 1000U//, 
três receberam estibogluconato de sódio. A associação de dor abdominal à elevação dos níveis séricos de amilase foi observada em 11 pacientes tratados com estibogluconato de sódio e em apenas três tratados com antimoniato de meglumina. Estes achados são compatíveis com os encontrados por outros autores que registraram elevação dos níveis de amilase e lipase em $97 \%$ de 17 pacientes portadores de diversas formas de leishmaniose, tratados com estibogluconato de sódio (Pentostan $\AA$ ). A pancreatite tóxica assintomática associada ao tratamento com os antimoniais pentavalentes foi relatada em pacientes com leishmaniose visceral tratados com antimoniato de meglumina a qual reverteu sem necessidade de interrupção do tratamento ${ }^{9}$. Os mecanismos pelos quais essas alterações não progridem, apesar da continuidade do tratamento ainda não foram esclarecidos. A maioria dos pacientes apresentou diminuição ou normalização de valores enzimáticos alterados sem descontinuar o tratamento. Entretanto, a importância da elevação observada em alguns pacientes justifica a monitorização dos níveis dessas enzimas. Os níveis elevados de amilase em pacientes assintomáticos, poderiam ser explicados pela produção desta enzima por outras glândulas, como por exemplo as salivares. Esta hipótese deverá ser estudada utilizando a caracterização isoenzimática de amilase.

A pesar de não terem sido detectadas alterações da função renal com o uso das duas drogas estudadas não se descarta a possibilidade de que tenham ocorrido disfunções discretas que não se refletiram nos níveis séricos de creatinina e uréia.

As principais alterações eletrocardiográficas observadas foram: aumento do intervalo QT (QTc); alteração da repolarização ventricular; alterações isquêmicas e extrassístoles bigeminadas, polimorfas e polifocais. Estas alterações, com exceção da última, têm sido freqüentemente descritas durante o tratamento com antimoniais pentavalentes (Glucantime $\AA$ ou Pentostan $\AA)^{12810}$. Apesar de não haver diferenças na freqüência das alterações eletrocardiográficas, nos dois grupos de tratamento, as alterações isquêmicas, que ocorreram em 19 pacientes, foram mais freqüentes com o uso do estibogluconato de sódio e a única alteração que levou a interrupção do tratamento, detectada no décimo dia, foi extrassístoles bigeminadas, polimorfas e polifocais em uma paciente do sexo feminino de 42 anos também tratada com esta droga. 0 mecanismo da toxicidade cardíaca atribuída aos antimoniais pentavalentes permanece obscuro; os nossos achados indicam que definitivamente é necessária a avaliação eletrocardiográfica durante o tratamento com antimoniais pentavalentes independentemente da presença de fatores que aumentem o risco de doença cardíaca. A associação de alterações enzimáticas e alterações eletrocardiográficas simultâneas ocorreu mais freqüentemente em pacientes tratados com estibogluconato de sódio. Este dado evidencia a diferença do potencial de toxicidade entre as duas drogas testadas.

A presença de antimonial trivalente nas ampolas de antimoniato de meglumina e estibogluconato de sódio tem sido incriminada na gênese da toxicidade destas drogas ${ }^{5}$. Esta possibilidade foi afastada medindo a quantidade de antimônio trivalente que foi desprezível nos lotes utilizados (dados não publicados). A osmolaridade foi significativamente mais elevada nas ampolas do estibogluconato de sódio (1274 mosM), do que nas do antimoniato de meglumina (783,2 mosM). Diferenças na osmolaridade dos antimoniais têm sido associadas com a toxicidade, mas os dados na literatura são controversos ${ }^{4} 1011$.

O estibogluconato de sódio BP $88 \AA$ é uma droga com maior toxicidade quando comparada ao antimoniato de meglumina e, portanto, não se mostrou uma droga alternativa para o tratamento das leishmanioses.

\section{AGRADECIMENTOS}

Às Doutoras Rosa Maria Veloso Rode e Eline Carvalho Pimentel de Oliveira que realizaram as provas de laboratório, à Fundação Nacional de Saúde, Ministério da Saúde por ceder os lotes de medicamento utilizados e ao Sr. Ednaldo Lima do Lago pela ajuda no desenvolvimento do trabalho de campo.

\section{REFERÊNCIAS BIBLIOGRÁFICAS}

1. Berman JD. Chemotherapy for leishmaniasis: Biochemical mechanisms, clinical efficacy and future strategies. Reviews of Infectious Diseases 10:560-586, 1988. 
2. Chulay JD, Spencer HC, Mugambi M. Eletrocardiographic changes during treatment of leishmaniasis with pentavalent antimony (sodium stibogluconate). The American Journal of Tropical Medicine and Hygiene 34:702-709, 1985.

3. Conselho Nacional de Pesquisa. Epidemiologia da Doença de Chagas. Objetivos e Metodologias de estudos longitudinais. Relatório Técnico no 1, Rio de Janeiro, 1974.

4. Costa JML. Estudo clínico epidemiológico de um surto epidêmico de Leishmaniose Tegumentar Americana em Corte de Pedra-BA. Tese de Mestrado, Universidade de Brasília, Brasília, DF, 1986.

5. Franco MA, Barbosa AC, Rath S, Dorea JG. Antimony oxidation states in antileishmanial drugs. The American Journal of Tropical Medicine and Hygiene 52:435-437, 1995.

6. Gasser RA, Magill AJ, Oster CN, Franke ED, Grogl M, Berman JD. Pancreatitis induced by pentavalent antimonial agents during treatment of leishmaniasis. Clinical Infectious Diseases 18:83-90, 1994

7. Hepburn NC, Siddique I, Howie AF, Beckett GJ, Hayes PC. Hepatotoxicity of sodium stibogluconate therapy for American Cutaneous Leismaniasis. Transactions of the Royal Society of Tropical Medicine and Hygiene 88:453$455,1994$.

8. Lacerda-Junior FS, Germiniani H, Caldas C, Mota S, Baranski MC. Estudo comparativo das alterações eletrocardiográficas determinadas pelo tratamento com antimoniais tri e pentavalentes. Revista do Instituto de Medicina Tropical de São Paulo 7:210-217, 1965.

9. Laguna F, Soriano V, González-LA JM. Misdiagnosis of pancreatitis in patients receiving treatment with pentavalent antimonial agents. Clinical Infectious Diseases 19:978-979, 1994.

10. Marsden PD. Pentavalent antimonials: old drugs for new diseases. Revista da Sociedade Brasileira de Medicina Tropical 18:187-198, 1985.

11. Merchan-Hamann E. Ensaio terapêutico com quatro dosagens de antimonial no tratamento da Leishmaniose Cutânea causada por Leishmania (Viannia) braziliensis. Tese de Mestrado. Universidade de Brasília, Brasília, DF, 1989.

12. Rosa AC, Cuba CC, Vexanat A, Barreto AC, Marsden PD. Predominance of Leishmania braziliensis braziliensis in the regions of Três Braços and Corte de Pedra, BahiaBrasil. Transactions Royal Society of Tropical Medicine and Hygiene 82:409-410, 1988.

13. Rose G, Blackburn H. Métodos de encuesta sobre enfermedades cardiovasculares. Organizacion Mundial de la Salud, Genebra, 1982.

14. Saldanha ACR, Romero GAS, Merchan-Hamann E, Magalhães AV, Macêdo VO. Estudo comparativo entre estibogluconato de sódio BP 88 , e antimoniato de meglumina no tratamento da leishmaniose cutânea: I. Eficácia e segurança. Revista da Sociedade Brasileira de Medicina Tropical 32:383-387, 1999. 